\title{
Properties of Anomalous and Type II Cepheids in the Small and Large Magellanic Clouds
}

\author{
Monika I. Jurkovic ${ }^{1,2, \star}$ and Martin A. T. Groenewegen ${ }^{3, \star \star}$ \\ ${ }^{1}$ Astronomical Observatory of Belgrade (AOB), Volgina 7, 11060 Belgrade, Serbia \\ ${ }^{2}$ Konkoly Observatory, Research Centre for Astronomy and Earth Sciences, Hungarian Academy of Sci- \\ ences, H-1121 Budapest, Konkoly Thege Miklós út 15-17, Hungary \\ ${ }^{3}$ Koninklijke Sterrenwacht van België (KSB), Ringlaan 3, B-1180 Brussels, Belgium
}

\begin{abstract}
The Small Magellanic Cloud (SMC) and Large Magellanic Cloud (LMC) give us the possibility to study individual variable star types in a new way. Literature data provide us with photometric information about objects from the ultraviolet to the infrared. Here we would like to show the results of our study of 335 Anomalous and Type II Cepheids in the SMC and LMC detected by OGLE. Using the code More of DUSTY (MoD), a modified version of the DUSTY radiative transfer code, and the assumption that our objects are at a known distance, luminosity and effective temperature were determined. From these data the Hertzsprung-Russell diagram of these objects was compared with the theoretical models. The radius and masses of the examined stars was estimated, too. In the end, we have given the period-luminosity relations for the Anomalous and Type II Cepheids.
\end{abstract}

\section{Introduction}

The goal of this work was to these stars using the physical effective temperatures $\left(T_{\text {eff }}\right)$ and luminosities $(L)$, instead of the previously used color-magnitude diagrams (CMD). This gives a direct comparison with the results of the evolutionary models. In the course of our research we have included the examination of infrared (IR) excess, and the role of binarity, as well. Our detailed results will be published in two recent papers $([3,4])$. We have extended our sample to the semi regular variables, also identified as long period variables (LPVs) in OGLE-III (see [5]).

Type II Cepheids (T2Cs) are low mass $\left(\sim 0.5-0.6 M_{\odot}\right)$ pulsating stars, and we follow the classification into subgroups given in the OGLE-III catalog: BL Her (BLH): 1-4 days; W Virginis (including peculiar W Virginis, (p)WVir: 4 - 20 days; RV Tauris (RVT): 20 - 70 days. They are useful distance indicators with $L$ and $T_{\text {eff }}$ between the RR Lyrae, Anomalous Cepheids (ACs), and Classical Cepheids (e.g., see [15]).

The ACs are also pulsating stars with pulsating periods ranging from 0.3 to 2.4 days, which overlaps in part with the RR Lyrae and BL Her types. Their average mass is $\sim 1.2 M_{\odot}$ ([1]). They pulsate in the fundamental mode and first overtone, and these subgroups form different period-luminosity $(P L)$ relations, nevertheless they seem not to reach into the Classical Cepheids region.

\footnotetext{
${ }^{\star}$ mojur@aob.rs

$\star \star$ martin.groenewegen@oma.be
} 
Table 1. The distribution of the T2Cs and ACs in the OGLE-III catalog of the SMC and LMC.

\begin{tabular}{ccccc}
\hline & BL Her & (p)W Vir & RV Tau & AC \\
\hline SMC & 17 & 17 & 9 & 6 \\
LMC & 64 & 97 & 42 & 83 \\
\hline
\end{tabular}

\section{The sample}

The 335 stars were taken from the OGLE-III catalog ([11-13]). For the distribution among the different types, see Table 1.

The spectral energy distributions (SEDs) for the 335 stars, as presented in [3], was constructed using the optical, infrared and near-infrared data from the catalogs available at Vizie ${ }^{1}$, which included: OGLE, EROS, MACHO, MCPS, DENIS, 2MASS, 2MASS 6X, IRSF, LMC Synoptic Survey, VMC Survey, WISE, Akari, IRAC.

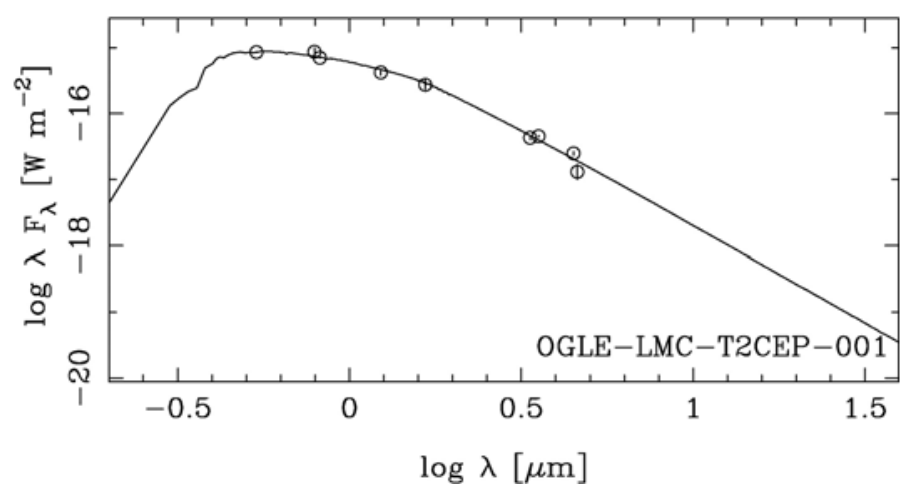

Figure 1. The SED for the OGLE-LMC-T2CEP-001, showing a normal distribution.

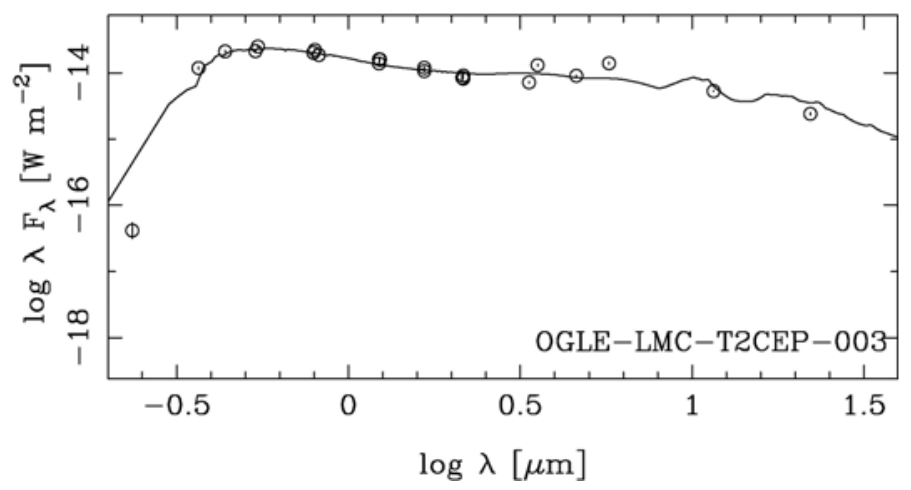

Figure 2. The SED for the OGLE-LMC-T2CEP-003 with a visible IR excess.

\footnotetext{
${ }^{1}$ http://vizier.u-strasbg.fr/viz-bin/VizieR
} 


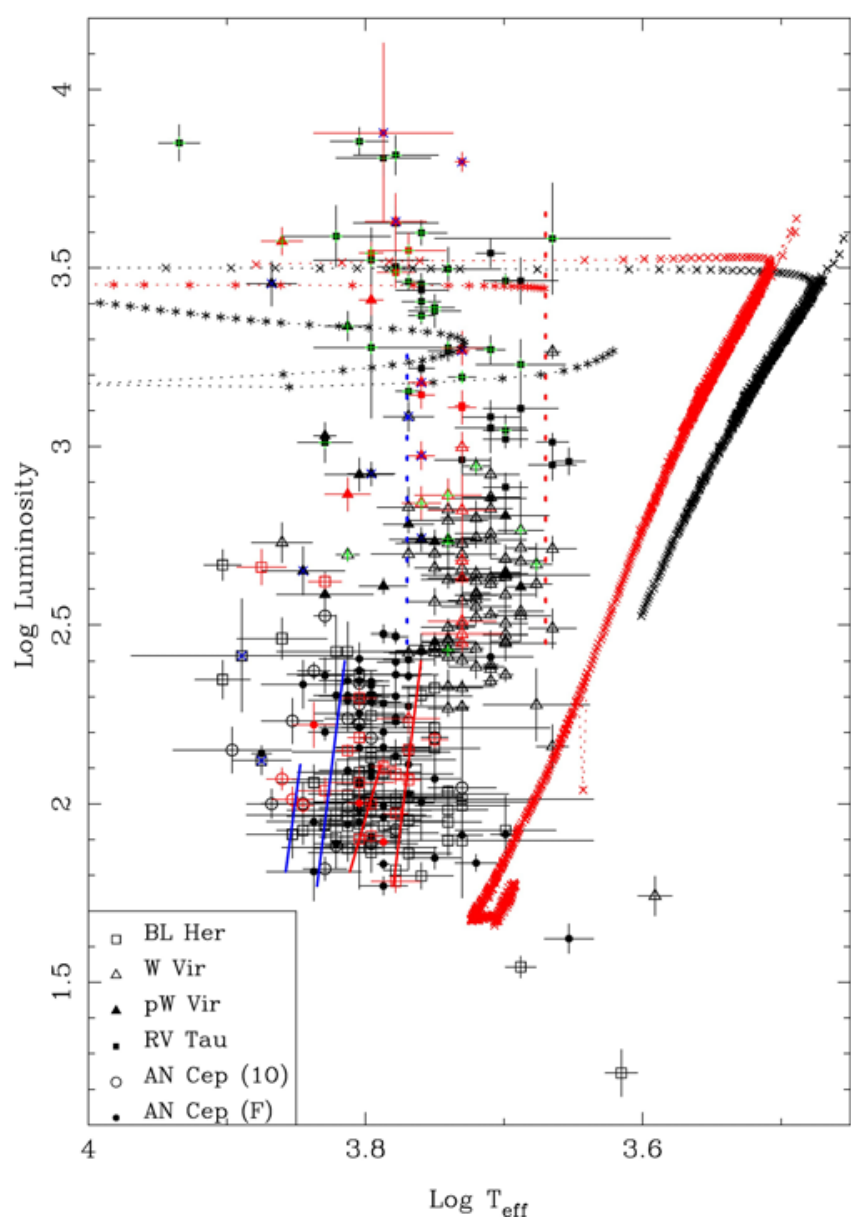

Figure 3. The HRD constructed from the modelled SEDs $T_{\text {eff }}$ and luminosity. Stars in the SMC are plotted in red. Stars with an IR excess are marked by a green plus-sign. Stars which show eclipsing or ellipsoidal variations according to OGLE are marked by a blue cross. The tracks plotted with $\times$ are the lowest initial mass tracks of [14] for the $\mathrm{LMC}\left(0.945 M_{\odot}\right)$ and $\mathrm{SMC}\left(0.89 M_{\odot}\right.$, in red). The final masses are 0.555 and $0.558 M_{\odot}$, respectively. The tracks plotted with an asterisk symbol are the lowest initial mass tracks of [10] for a metallicity of $Z=0.01$ (1.0 $M_{\odot}$, current mass $\left.0.534 M_{\odot}\right)$ and $Z=0.001\left(0.9 M_{\odot}\right.$, current mass $0.536 M_{\odot}$, in red). In both tracks every tick mark represents 500 years of evolution. The blue and red edges of the fundamental (FU) mode instability strip of BLH (between $\log L \sim 1.81-2.1$, for a mass of $0.65 M_{\odot}$ ) and FU ACs (between $\log L \sim 1.77-2.4$ ) are indicated by the solid line (see text). The vertical dotted lines indicate the location of most of the variables at higher luminosity (see text). Taken from [3], with the permission of the authors.

The dust radiative transfer code More of DUSTY was used ([2]), which is an extension of DUSTY ([6]). The loop over $T_{\text {eff }}$ ran from 2900 to $10000 \mathrm{~K}$. A $\chi^{2}$ fit was done to search for the model that fitted the data the best, giving us the $T_{\text {eff }}$ and $L$ for each object. MARCS model atmospheres were used. The distance for the LMC and SMC were fixed at the following values, $d_{\mathrm{LMC}}=50 \mathrm{kpc}$ and $d_{\mathrm{SMC}}=61 \mathrm{kpc}$. A single $\mathrm{E}(\mathrm{B}-\mathrm{V})=0.15$ was adopted for all the stars. For the stars where IR excess was detected we fitted a model including dust. Examples of the fitted SEDs are in Figure 2. 


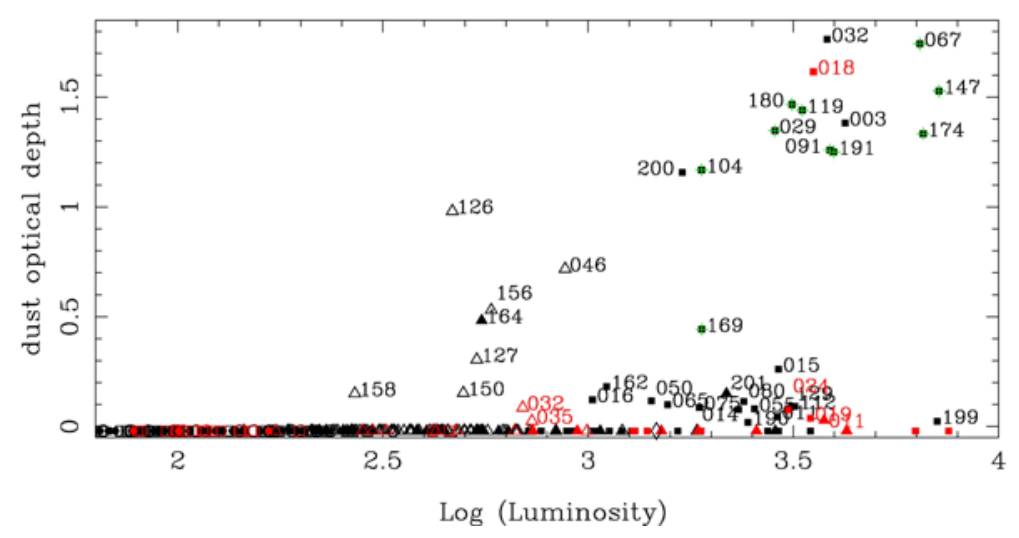

Figure 4. Dependence of the derived dust optical depth against $\log L$. Stars in the SMC are plotted in red. Stars with an IRS spectrum are marked by a green plus-sign. Stars with a detectable IR excess are labelled by their identifier. Taken from [3], with the permission of the authors.

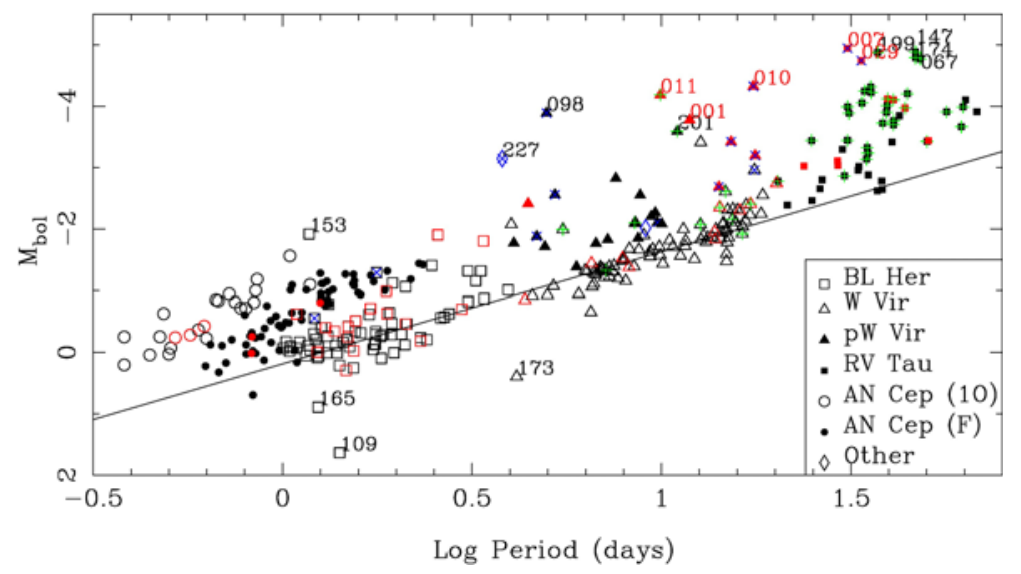

Figure 5. The bolometric magnitude vs. $\log P P L$ relation tor the T2Cs and ACs in the SMC and LMC. Taken from [4] with the permission of the authors.

\section{HRD}

Figure 3 is the physical Hertzsprung-Russell diagram (HRD) of the T2Cs and ACs in the SMC and LMC. For the detailed description of the symbols, see the caption and [3].

\section{The IR excess}

Among the examined stars we have found that $\sim 60 \%$ of RVT, and $\sim 10 \%$ of the WVir (including the peculiar WVir), show IR excess; see Figure 4. The distributions among subtypes looks like this:

- ACEP: 0/76;

- BLH: 0/81; 
- (p)WVir: 8/90 for WVir and 3/24 for pWVir;

- RVT: 30/52.

It was not surprising to find IR excess among the RVT stars, because they are thought to be pAGB objects. What was surprising is the detection of IR excess among the WVir stars below luminosities predicted by single star evolutionary tracks. This IR excess was independently found by [7-9] to be dusty post-RGB stars and possibly related to binary evolution.

\section{The PL relation}

Using the gathered data we were able to construct the bolometric magnitude vs. $\log P P L$ relation for the T2Cs and ACs in the sample; see Figure 5 and [4].

\section{Conclusion}

We have determined the luminosities and effective temperatures of the sample of $335 \mathrm{~T} 2 \mathrm{Cs}$ and ACs in the SMC and LMC. This investigation provided us with data to create the HRD that could be directly compared to the evolutionary model results.

We have derived the $P L$ relation for all the stars in the sample.

We have found infrared excess in $10 \%$ of the W Vir stars and $60 \%$ of the RV Tau stars. The findings by [9] of the existence of (pulsating) stars that have a lower luminosity value than the luminosity predicted if they would have been evolving as a single star, and that they show IR excess connected to binarity, are also confirmed by us.

For further details, please, look into our articles [3] and [4].

Acknowledgments: M.I.J. acknowledges financial support from the Ministry of Education, Science and Technological Development of the Republic of Serbia through the project 176004, and the Hungarian National Research, Development and Innovation Office through NKFIH K-115709.

\section{References}

[1] Fiorentino, G., \& Monelli, M., A\&A, 540, A102 (2012)

[2] Groenewegen, M. A. T., A\&A, 543, A36 (2012)

[3] Groenewegen, M. A. T., \& Jurkovic, M. I., A\&A, in press (arXiv:1705.00886) (2017a)

[4] Groenewegen, M. A. T., \& Jurkovic, M. I., A\&A, in press (arXiv: 1705.04487) (2017b)

[5] Groenewegen, M. A. T., \& Jurkovic, M. I., in these proceedings

[6] Ivezić, Ž., Nenkova, M., \& Elitzur, M., DUSTY user manual, (arXiv:astro-ph/9910475) (1999)

[7] Kamath, D., Wood, P. R., \& Van Winckel, H., MNRAS, 439, 2211 (2014)

[8] Kamath, D., Wood, P. R., \& Van Winckel, H., MNRAS, 454, 1468 (2015)

[9] Kamath, D., Wood, P. R., Van Winckel, H., \& Nie, J. D., A\&A, 586, L5 (2016)

[10] Miller Bertolami, M. M., A\&A, 588, A25 (2016)

[11] Soszyński, I., Udalski, A., Szymański, M. K., et al., AcA, 58, 293 (2008)

[12] Soszyński, I., Poleski, R., Udalski, A., et al., AcA, 60, 17 (2010)

[13] Soszyński, I., Udalski, A., Szymański, M. K., Kubiak, M., Pietrzyñski, G., Wyrzykowski, Ł., Ulaczyk, K., \& Poleski, R., et al., AcA, 60, 91 (2010)

[14] Vassiliadis, E \& Wood, P. R., ApJ, 413, 641 (1993)

[15] Wallerstein, G., PASP, 114, 689 (2002) 